\title{
Pain from a Bullet Lingers on: An Uncommon Case of Lead Toxicity
}

\author{
Albin Abraham ${ }^{a}$ Jaspreet Singh $^{b} \quad$ Paul Mustacchia $^{b}$ \\ Kaleem Rizvon ${ }^{\text {b }}$ \\ Departments of ${ }^{a}$ Internal Medicine and ${ }^{b}$ Gastroenterology, Nassau University \\ Medical Center, East Meadow, N.Y., USA
}

\section{Key Words}

Lead toxicity $\cdot$ Intra-articular bullets $\cdot$ Abdominal pain $\cdot$ Chelation therapy

\begin{abstract}
Lead toxicity from a retained bullet as a cause for abdominal pain is rarely considered. Given its unpredictable latent period and nonspecific clinical symptoms, such cases are difficult to diagnose but may be fatal if unrecognized. We present the case of a 48-year-old man who presented with complaints of abdominal pain, weight loss and constipation. His past history was significant for a gunshot wound to the left hip about 20 years before. Radiographic studies confirmed the same with the presence of numerous intra-articular bullet fragments and a calcified hemarthrosis surrounding the left femoral head. Blood lead levels were elevated following which the patient was started on chelation therapy with succimer which resulted in symptomatic improvement. The aim of this paper is to highlight the importance of considering lead toxicity from a retained bullet as a cause of abdominal pain and to review the relevant literature.
\end{abstract}

\section{Introduction}

Due to its highly desirable properties such as low melting point, malleability, durability and low costs, lead has become widely distributed and mobilized in the environment. However, lead is also a potent toxin and human exposure to this metal has become a health problem of global dimensions. The Adult Blood Lead Epidemiology and Surveillance (ABLES) program estimates the prevalence of elevated blood lead levels in the Unites States between 2005 and 2007 to be around 7.8 per 100,000 [1]. The majority of adult elevated blood lead levels come from work exposure such as manufacturing of batteries, pigments, ammunitions and car radiators. Leaded gasoline 
continues to be used in many parts of the world, which contributes to the atmospheric burden.

Lead poisoning from intra-articular bullets has been recognized since 1867 [2]. The diagnosis in most cases is however difficult and often delayed given the vague and transient symptoms and the unpredictable time interval to the onset of such symptoms. Lead toxicity may manifest as abdominal pain, vomiting, constipation, fatigue, arthralgias, headache, decreased libido, hypertension and anorexia. Blood lead levels may fluctuate with rapid bone growth, pregnancy, lactation or stressful events such as sepsis or surgery. The latent period from exposure to onset of symptoms has been reported to range from 2 days to 52 years [3]. Treatment entails chelation therapy and definitive surgical removal of the bullet. To reduce the disease burden caused by the exposure to lead, it is important to determine the groups at highest risk and the main pathways of exposure that contribute to health impacts.

\section{Case Report}

A 48-year-male presented to our hospital with complaints of abdominal pain and change in bowel habits. He described the pain as being constant for the past 3 months, located in his mid abdomen, dull and aching in nature, moderate to severe in intensity with no radiation, aggravating or relieving factors. He also complained of constipation during the same period associated with a weight loss of 30 pounds. Review of systems was unremarkable for fever, chills, nausea, vomiting, headache, visual complaints, chest pain, shortness of breath, odynophagia, dysphagia, blood per rectum or black stools, joint pains, tingling, numbness, paresthesias, muscle weakness or skin rash. The patient had emigrated from Guatemala 15 years earlier. He had a recent workup in his native country for the abdominal pain which was unremarkable and it was recommended that he be follow up in the United States. His past medical history was significant for diabetes mellitus type 2, hypertension and hyperlipidemia. The patient also had suffered a gunshot injury to his left hip more than 20 years before. Home medications included candesartan, nebivolol, celecoxib, glimepiride and sitagliptin. He denied the use of alcohol, tobacco or illicit drugs. He had been employed as a plumber for more than 10 years in a construction company taking care of old houses.

On physical examination the patient appeared comfortable and in no apparent distress. Vital signs on admission revealed a blood pressure of $170 / 100 \mathrm{~mm} \mathrm{Hg}$, a pulse rate of $68 \mathrm{bpm}$, a respiratory rate of 14 per minute and a temperature $98.3^{\circ}$ Fahrenheit. The patient had no conjunctival pallor or scleral icterus, oral thrush, gum line or lymphadenopathy. The cardiovascular and respiratory system were unremarkable. The abdomen was soft, nondistended with mild periumbilical tenderness but no rebound tenderness, rigidity or guarding. There was no organomegaly or any stigmata of chronic liver disease. There were no masses or blood on digital rectal examination. There was limitation to the range of movement in the left hip joint.

Laboratory investigations revealed a hemoglobin of $10.4 \mathrm{~g} / \mathrm{dl}$ and a hematocrit of $30.4 \%$ with mean corpuscular volume $88.9 \mathrm{fl}$, mean corpuscular hemoglobin $30.3 \mathrm{pg}$ and red cell distribution width $14.4 \%$. Iron studies showed iron to be $111 \mu \mathrm{g} / \mathrm{dl}$, total iron binding capacity $210 \mu \mathrm{g} / \mathrm{dl}$, transferring saturation $53 \%$ and ferritin $613 \mathrm{ng} / \mathrm{ml}$. Liver-related tests, blood chemistry and amylase as well as lipase levels were within normal limits. Computed tomography of the abdomen and pelvis confirmed the gunshot wound to the left hip with a calcified hemarthrosis surrounding the left femoral head (fig. 1, fig. 2). X-ray of the left hip showed advanced degenerative changes to the joint with numerous tiny intra-articular bullet fragments (fig. 3 ). Colonoscopy results revealed spastic colon with no other abnormality. The patient's prior workup was reviewed and showed normal upper endoscopy, normal TSH, uric acid and urine porphobilinogen levels. Given the history of the gunshot wound confirmed by computed tomography scan and the occupation of the patient, a blood lead level was sent which was reported back as being $63.4 \mu \mathrm{g} / \mathrm{dl}$ (elevated blood lead levels are defined as levels $>25 \mu \mathrm{g} / \mathrm{dl}$ ). The peripheral blood smear which was later viewed showed basophilic stippling. The Regional Toxicology Center and Department of Health were contacted and the patient was started on a 19-day oral succimer chelation therapy. His abdominal pain abated on the fourth day of therapy 
with improvement also in his bowel movements. He was later discharged with follow-up appointments at outpatient clinics and was advised not to join work till his next clinic visit.

On a follow-up visit in our outpatient clinic 2 weeks after discharge, the patient was symptom-free and his blood lead level was $43.5 \mu \mathrm{g} / \mathrm{dl}$. His hemoglobin had also improved to $12.1 \mathrm{~g} / \mathrm{dl}$. He later underwent total left hip arthroplasty with removal of the bullet. His blood lead level 1 week after the surgery was $39.7 \mu \mathrm{g} / \mathrm{dl}$. The patient was doing well at the 6 -month follow-up evaluation.

\section{Discussion}

Lead has been mined for thousands of years and was once prized as one of the most valuable elements on earth. However in the past century due to tremendous scientific data available, the toxic effects of lead on the human body have been demonstrated. Tanquerel des Plances described the clinical course of workers, primarily painters, who developed lead colic in 1839, leading to the initial interest in the illness caused by lead poisoning. Lead poisoning is now considered one of the most common diseases of environmental origin. The blood lead levels of Americans as monitored by the National Health and Nutrition Examination Survey (NHANES) indicate that the mean blood lead levels have declined by more than $80 \%$, from $12.8 \mu \mathrm{g} / \mathrm{dl}$ (in 1976-1980) to a current level of $2 \mu \mathrm{g} / \mathrm{dl}$. Blood lead levels begin to rise in adolescence through adulthood, reflecting occupational exposure, and then rise again in the elderly, reflecting remobilization of lead as bone resorption occurs. Though the normal lead levels in the past were considered to be $<40 \mu \mathrm{g} / \mathrm{dl}$, the presence of subclinical effects of lead at lower levels argues for defining lead intoxication as blood lead levels $>25 \mu \mathrm{g} / \mathrm{dl}$ [4].

The most common sources of lead toxicity are lead paint, air polluted with industrial and automobile fumes, contaminated water and soil and occupational exposures as in plumbers, miners, welders and battery manufacturers. Among the rarer causes, lead poisoning due to retained bullets has often been described in the literature. Bronvin [5] recorded the first such case in the modern medical literature in 1867 [6]. Ellis [7] published the first English language report of a possible association between retained bullets and lead toxicity in 1874. Our patient had two potential etiologies of lead toxicity: his occupation and the retained bullet in his left hip.

Many factors affect the risk of lead toxicity from a retained bullet: location, fragmentation, number and lead content of the bullets [8]. Lead bullets in muscle or bone generally do not result in toxicity as the tissue surrounding the foreign body creates a fibrous capsule and prevents the dissolution of lead into the blood circulation [9]. However bullets lodged in joints or in the spinal cord canal are in constant contact with the acidic synovial fluid or cerebrospinal fluid. The metallic lead tends to dissolve in the acidic media, thereby promoting its absorption, distribution and toxic effects in the target tissues and organs. In addition the resultant arthritic inflammation further increases vascularity to the joint, accelerating the rate of systemic absorption. Friction within a mobile joint can also hasten fragmentation of the bullet [5]. McQuirter et al. [10] reported that retained lead bullets are often fragmented (69.2\%) and that blood lead levels increase by $25.6 \%$ with fragmentation mainly by increased surface area of absorption. When fragmentation is combined with a torso bone fracture there is a further increase in blood lead levels of $29.5 \%$. This can be explained by the fact that the torso contains several body cavities which lead fragments are exposed to after a penetrating injury. The development of pseudocysts near the bone following a fracture 
containing finely dispersed lead fragments has also been described [11]. Another variable which determines the burden of lead-related toxicity is the lead content in the projectile. Bullets and shotgun pellets encountered by US civilians are typically made from a lead alloy or are clad bullets with a lead core. Lead bullets which lack a metal cover have a greater surface area of lead exposed for dissolution. The variables mentioned above can also contribute to the discrepancies in the reported onset of symptoms from the initial insult.

Once in circulation, lead diffuses into the soft tissues including the liver, kidneys, bone marrow and brain. After a period of 1-2 months lead diffuses into the bone where it is incorporated into the hydroxyapatite lattice. Here it is presumed to be inert and nontoxic. However conditions that cause bone mobilization like pregnancy, bed rest, medications and advancing age can result in mobilization of lead leading to toxicity. Symptoms related to lead toxicity can include peripheral neuropathy, fatigue, headaches, memory loss, seizures, hypertension, anemia, gingival lead lines, muscle and joint aches. Gastrointestinal manifestations of lead poisoning can include abdominal colic, anorexia, weight loss and altered bowel habits. The pathophysiology of these symptoms is unclear. Dasani and Kawanishi [12] suggested that lead may induce intestinal spasms and interfere with the receptors of the gastrointestinal tract, leading to anorexia. Because of lead's large volume of distribution, the blood lead level is a relatively poor indicator of the total lead burden. Nonetheless, since it is easy to obtain and provides useful information it is still extremely valuable. X-ray fluorescence is a new and promising test that measures the skeletal lead load. Studies show that X-ray fluorescence may be more accurate than blood lead level measurement. Other laboratory tests that may be useful in evaluation and management include complete blood count that may show anemia, peripheral blood smear that may show basophilic stippling, renal function tests and $\delta$-aminolevulinic acid dehydratase activity [4].

Though definitive management involves surgery to remove the lead fragments, it is not usually indicated as the initial therapy for patients with lead toxicity. The patient should first be stabilized and the lead burden reduced by chelation therapy. Surgery may mobilize bone lead stores by bone or tissue manipulation, stress, physical inactivity or metabolic imbalances and thereby result in worsening of lead toxicity even leading to death. Parenteral agents used for chelation therapy include dimercaprol and calcium disodium ethylene diamine tetraacetic acid (CaNa2EDTA). The two most commonly used oral agents are d-penicillamine and succimer. Succimer as was administered to our patient is available in $100 \mathrm{mg}$ capsules. The current treatment protocol is administration of $10 \mathrm{mg} / \mathrm{kg}$ per dose. For the first 5 days it is given every $8 \mathrm{~h}$, and for the next 14 days every $12 \mathrm{~h}$. Adverse reactions include rash, flu-like symptoms and elevated liver transaminases and can be seen in $5 \%$ of patients $[3,4,8,11]$.

McQuirter et al. [10] reported blood lead levels $\geq 10 \mu \mathrm{g} / \mathrm{dl}$ in $38.1 \%$ and levels $\geq 20 \mu \mathrm{g} / \mathrm{dl}$ in $11.8 \%$ at 3 months post injury. This highlights the need for surveillance in patients with retained bullets after gunshot wounds. First, they should have a lead level at admission for baseline records. Second, they should have their levels retested before discharge or 2 weeks after injury. The patients should be counseled to avoid further exposures to lead if occupational exposure is a cause of concern at discharge. Finally they should have lead levels checked monthly till 3 months post injury and then 1 year post injury. Thereafter they should be advised to obtain yearly blood lead levels. 
In conclusion, lead toxicity should be excluded in patients presenting with nonspecific symptoms such as abdominal pain and with a history of a gunshot wound. The health implications are tremendous as there are an estimated 1-2 million gunshot wound survivors with retained bullets in the United States, with thousands being added yearly.

\section{Disclosure Statement}

The authors have no funding or conflicts of interest to disclose.

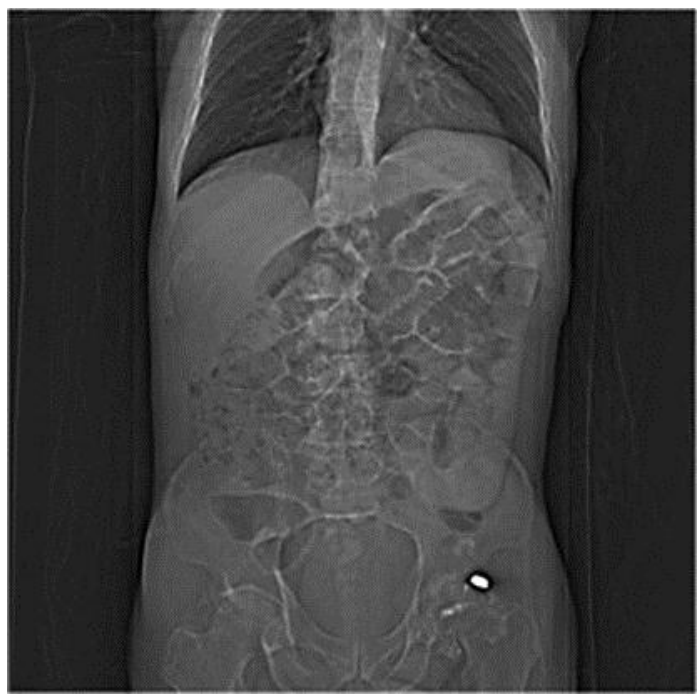

Fig. 1. Computed tomography scan showing a bullet in the left hip with a calcified hemarthrosis surrounding the left femoral head.

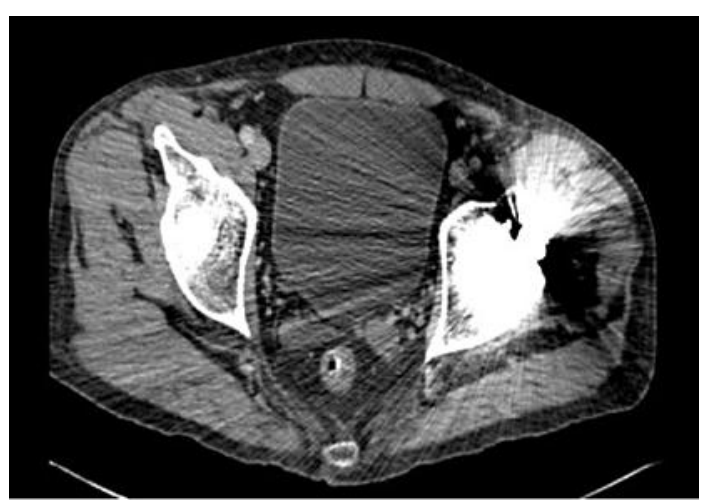

Fig. 2. Computed tomography scan showing the bullet in the left hip. 


\begin{tabular}{r|l|l|l}
$\begin{array}{r}\text { Case Reports in } \\
\text { Gastroenterology }\end{array}$ & $\begin{array}{l}\text { Case Rep Gastroenterol 2012;6:243-248 } \\
\text { DOI: 10.1159/000338843 }\end{array}$ & $\begin{array}{l}\text { Published online: } \\
\text { May 8, 2012 }\end{array}$ & $\begin{array}{l}\text { @ 2012 S. Karger AG, Basel } \\
\text { ISSN 1662-0631 } \\
\text { www.karger.com/crg }\end{array}$ \\
\hline
\end{tabular}

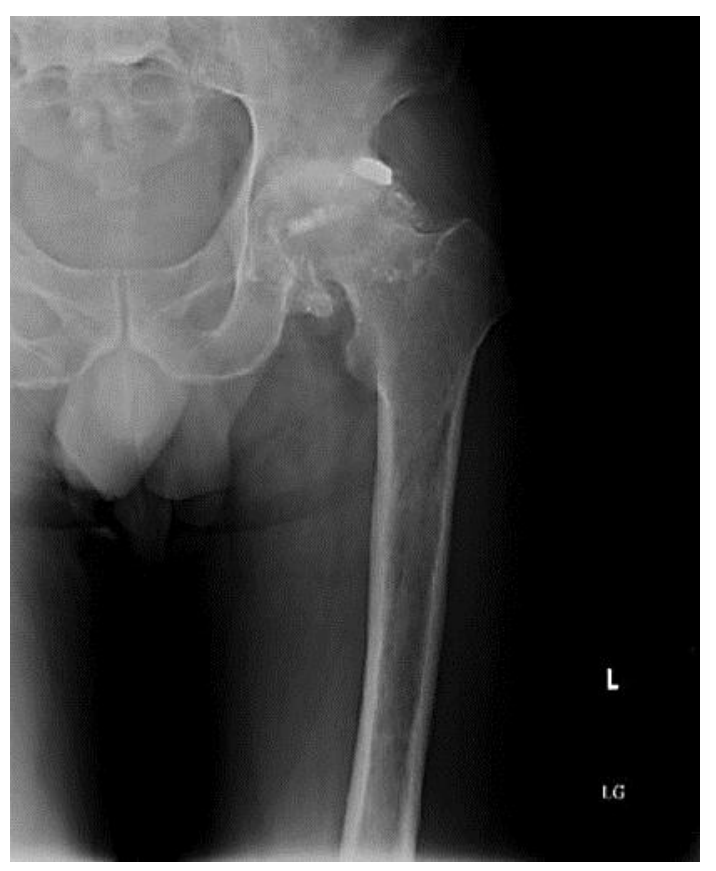

Fig. 3. X-ray of the left hip showing advanced posttraumatic arthritic changes including tiny intra-articular bullet fragments.

\section{References}

1 Centers for Disease Control and Prevention (CDC): Adult blood lead epidemiology and surveillance United States, 2005-2007. MMWR Morb Mortal Wkly Rep 2009;58:365-369.

-2 DeMartini J, Wilson A, Powell JS, Powell CS: Lead arthropathy and systemic lead poisoning from an intraarticular bullet. AJR Am J Roentgenol 2001;176:1144.

-3 Coon T, Miller M, Shirazi F, Sullivan J: Lead toxicity in a 14-year-old female with retained bullet fragments. Pediatrics 2006;117:227-230.

4 Shannon MW: Lead; in Shannon MW, Borron SW, Burns MJ (eds): Haddad and Winchester's Clinical Management of Poisoning and Drug Overdose, ed 4. Philadelphia, Saunders Elsevier, 2007, pp 11291146.

5 Bronvin S: Etiologie de la colique de plomb. Union Med 1867;3:89.

6 Dillman RO, Crumb CK, Lidsky MJ: Lead poisoning from a gunshot wound. Report of a case and review of the literature. Am J Med 1979;66:509-514.

7 Ellis C: A case of probable lead poisoning, resulting fatally from a bullet lodged in the knee joint twelve years previously. Boston Med Surg J 1874;91:472.

8 Akhtar A, Funnye A, Akanno J: Gunshot-induced plumbism in an adult male. J Natl Med Assoc 2003;95:986-990.

-9 Madureira P, Capitani E, Vieiria R: Lead poisoning after gunshot wound. Sao Paulo Med J 2000;118: 78-80.

10 McQuirter JL, Rothenberg SJ, Dinkins GA, Kondrashov V, Manalo M, Todd AC: Change in blood lead concentration up to 1 year after a gunshot wound with a retained bullet. Am J Epidemiol 2004;159: 683-692.

11 Linden MA, Manton WI, Stewart RM, Thal ER, Feit H: Lead poisoning from retained bullets: pathogenesis, diagnosis and management. Ann Surg 1982;195:305-313.

12 Dasani BM, Kawanishi H: The gastrointestinal manifestations of gunshot-induced lead poisoning. J Clin Gastroenterol 1994;19:296-299. 